Harriet C. Thoeny

Karl T. Beer

Peter Vock

Richard H. Greiner

\section{Ear pain in patients with oropharynx carcinoma: how MRI contributes to the explanation of a prognostic and predictive symptom}

Received: 22 October 2003

Revised: 11 March 2004

Accepted: 5 April 2004

Published online: 1 May 2004

(C) Springer-Verlag 2004

H. C. Thoeny $(-$ P. Vock Department of Diagnostic Radiology,

Inselspital,

University of Bern,

Freiburgstrasse 10, 3010 Bern, Switzerland e-mail: harriet.thoeny@insel.ch,

thoeny_harriet@hotmail.com

Tel.: +41-31-6322939

Fax: +41-31-6324873

K. T. Beer · R. H. Greiner

Department of Radiation Oncology,

Inselspital,

University of Bern,

Freiburgstrasse 10, 3010 Bern, Switzerland

\begin{abstract}
Reflex otalgia is a predictive and prognostic parameter for local control in patients with oropharynx carcinoma. Can a morphologic correlate of this important symptom be detected by MRI? Thirty-six patients were prospectively evaluated by MRI before radical radiotherapy. Sixteen patients had reflex otalgia; 20 did not. The oropharynx and adjacent regions were analyzed. Alteration was defined as effacement of anatomical structures, signal alteration or enhancement after contrast medium administration. The $\chi^{2}$-test was used to compare categorical parameters. In patients with reflex otalgia, alteration of the following structures innervated by the glossopharyngeal nerve were found significantly more often: nasopharynx, hard palate, superior constrictor pharyngis muscle, palatine tonsil, palatopharyngeus muscle, palato-
\end{abstract}

glossus muscle, stylopharyngeus muscle, hyoglossus muscle and preepiglottic space. No difference was found for the muscles of mastication, levator and tensor veli palatini muscles, styloglossus muscle, genioglossus muscle, intrinsic muscles of the tongue, digastric muscles, mucosal surface of the lateral and posterior pharyngeal wall, uvula, valleculae, parapharyngeal space and larynx. An alteration of structures innervated by the glossopharyngeal nerve was visualized on MRI significantly more often when reflex otalgia was present. Involvement of structures innervated by other cranial nerves did not show the same association with ear pain.

Keywords Head and neck neoplasms · Oropharynx · Magnetic resonance imaging . Earache

\section{Introduction}

Reflex otalgia is a common clinical symptom of patients with ENT diseases and can be caused by a variety of pathologies in the nasopharynx, oropharynx and hypopharynx. As a clinical symptom of oropharyngeal carcinoma, it is an important predictive and prognostic parameter of local control [1]. Patients with the symptom characteristically locate the pain in the middle ear, although only structures of the oropharynx are involved [1-4]. The anatomical basis of reflex otalgia, in general, is axonal connections between two cranial nerves via anastomoses. These connections exist for various cranial nerves innervating the nasopharynx (trigeminal nerve) and hypopharynx (vagal nerve) [3-6]. The underlying mechanism of the referred pain is an irritation of sensory nerve fibers, in this case of the glossopharyngeal nerve with connections to the middle ear via Jacobson's nerve (Fig. 1).

In order to evaluate the association of infiltration of structures innervated by the glossopharyngeal nerve as seen on imaging studies and reflex otalgia, we studied 36 consecutive patients with oropharynx carcinoma. 
Table 1 Patient characteristics

\begin{tabular}{llll}
\hline & Reflex otalgia & No reflex otalgia & All patients \\
\hline Number & 16 & 20 & 36 \\
Female; male & $12 ; 4$ & $2 ; 18$ & $14 ; 22$ \\
Smoker & 16 & 15 & 31 \\
Alcohol abuse & 15 & 15 & 30 \\
Age, median, range & 57 y (41-67) & 62 y $(34-86)$ & 60 y (34-86) \\
\hline
\end{tabular}

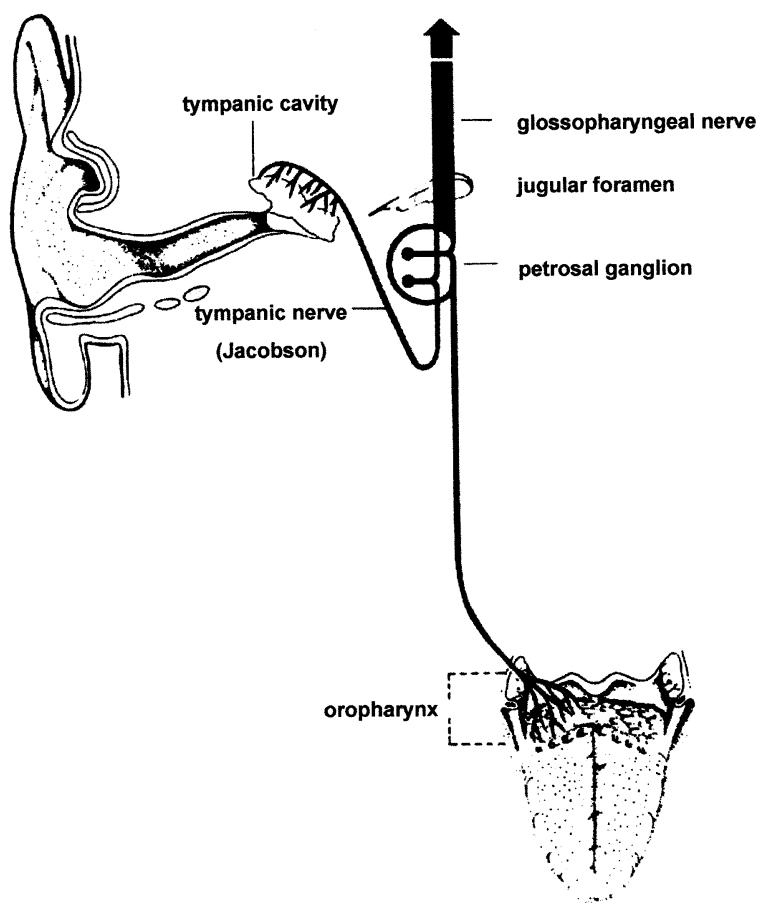

Fig. 1 Schematic illustration of the connection of the IX cranial nerve to the middle ear via Jacobson's anastomosis

\section{Materials and methods}

Patients

Thirty-six consecutive patients (14 women, 22 men) with a median age of 60 years (range, 34-86 years) undergoing a diagnostic work-up of newly diagnosed and biopsy-proven squamous cell carcinoma of the oropharynx were studied prospectively (Table 1). Sixteen patients suffered from reflex otalgia; 20 did not. The initial pretherapeutic MRI study was evaluated, and oral informed consent to re-examine the studies was obtained.

\section{MRI study}

All MRI studies were performed on a 1.5-T MR unit (18 MR examinations on a Magnetom Vision, Siemens, Erlangen, Germany and 18 on a Signa, GE, Milwaukee, USA) with a phased-array neck coil from the skull base to the upper mediastinum. Axial, coronal and/or sagittal spin-echo T1-weighted sequences and axial fast (turbo-) spin-echo PD/T2-weighted sequences were obtained on all patients. After intravenous (i.v.) administration of $0.2 \mathrm{mmol} / \mathrm{kg}$ of body weight of meglumin-gadoterat (Dotarem;
Guerbet, Roissy, France), axial, coronal and/or sagittal spin-echo T1-weighted series were acquired with and without fat saturation. A section thickness of 3-4 $\mathrm{mm}$ and an intersection gap of $1 \mathrm{~mm}$ were used. The field of view was $20-24 \mathrm{~cm}$. T1-weighted images (repetition time/echo time $=340-726 \mathrm{~ms} / 10-16 \mathrm{~ms}$ ), and fast (turbo-) spin-echo proton-density (PD)-weighted images (2,911$4,500 \mathrm{~ms} / 16-19 \mathrm{~ms})$ as well as T2-weighted images (2,911-4,500 $\mathrm{ms} / 80-119 \mathrm{~ms}$ ) were performed with a matrix of 154-256 $\times$ 256-512, and two to three signals were acquired. Repetition and echo times were identical in precontrast and postcontrast imaging.

\section{Study analysis}

We used the Handbook of Head and Neck Radiology by Ric Harnsberger [7] for definition of the areas innervated by the different cranial nerves. Interestingly, different anatomical textbooks describe a slightly different innervation by the cranial nerves [7, 8]. Alterations of the following anatomical structures were considered: within the oropharynx, the soft palate, superior constrictor pharyngis muscle, uvula, palatine tonsil, palatopharyngeus muscle, palatoglossus muscle, mucosal surface of the lateral and posterior pharyngeal wall, glossotonsillar sulcus, base of tongue, glossoepiglottic fold, pharyngoepiglottic fold and stylopharyngeus muscle, and at adjacent regions, the maxilla, the retromolar trigone, the hard palate, the muscles of mastication, the digastric muscles, styloglossus muscle, hyoglossus muscle, genioglossus muscle, mylohyoid muscle, geniohyoid muscle, intrinsic muscles of the tongue, submandibular and sublingual space, tensor and levator veli palatini muscles, skull base, the prevertebral space, the retropharyngeal and parapharyngeal spaces, the nasopharynx, the hypopharynx, the preepiglottic space and vocal cords.

Alteration was defined as an effacement of the above-mentioned anatomical structures, as a signal alteration in the different pulse sequences or an enhancement after administration of the contrast agent. It included both tumor infiltration and adjacent edema if present, because it was impossible to differentiate these two entities radiologically. The signal intensity of the tumor was noted with reference to the signal intensity of the adjacent muscles. Qualitative signal change was assessed by visual comparison with the surrounding muscles. The images were analyzed in consensus by two radiologists who knew the diagnosis of oropharyngeal carcinoma, but were blinded to the presence or absence of reflex otalgia. For tumor classification, TNM categories according to the 1997 classification were used [9].

\section{Statistics}

Categorical variables were compared by a two-tailed $\chi^{2}$-test. A $P$ level of less than 0.05 was considered to be statistically significant. SPSS (version 10.0, Wacker Drive, Chicago, Ill) was used for the calculations. 


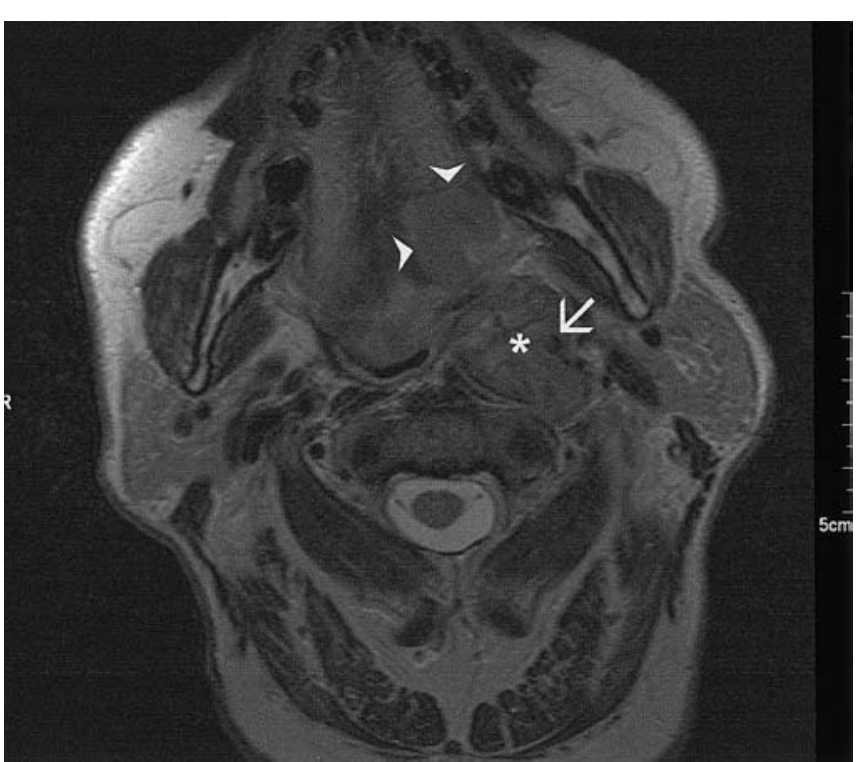

Fig. 2 Axial T2-weighted spin-echo MR-image (TR/TE: 4,000 ms/ $97 \mathrm{~ms}$ ) of a 63-year-old male patient suffering from ear pain. Leftsided soft tissue mass postero-laterally in the tongue base (arrowheads), corresponding to a squamous cell carcinoma. Large retropharyngeal adenopathy (asterisk) displacing the internal carotid artery antero-laterally (arrow)

Table 2 TNM classification of patients with and without reflex otalgia

\begin{tabular}{lllllc}
\hline & N0 & N1 & N2 & N3 & All \\
\hline T1 & $0 / 1$ & $0 / 0$ & $0 / 1$ & $0 / 0$ & $0 / 2$ \\
T2 & $0 / 0$ & $0 / 1$ & $0 / 3$ & $0 / 0$ & $0 / 4$ \\
T3 & $0 / 2$ & $2 / 0$ & $5 / 4$ & $0 / 1$ & $7 / 7$ \\
T4 & $1 / 1$ & $1 / 2$ & $6 / 1$ & $1 / 3$ & $9 / 7$ \\
All & $1 / 4$ & $3 / 3$ & $11 / 9$ & $1 / 4$ & $16 / 20$ \\
\hline
\end{tabular}

Numbers indicate patients in each TNM category with/without reflex otalgia, respectively

Table 3 Tumor localization

\begin{tabular}{lccr}
\hline & Reflex otalgia & No reflex otalgia & All \\
\hline Tonsil & 8 & 8 & 16 \\
Base of tongue & 6 & 8 & 14 \\
Soft palate & 2 & 4 & 6 \\
All & 16 & 20 & 36 \\
\hline
\end{tabular}

\section{Results}

No association was found between reflex otalgia and age, smoking or drinking habits. More female patients suffered from reflex otalgia (Table 1). Both groups were similar in tumor classification according to the TNM categorization

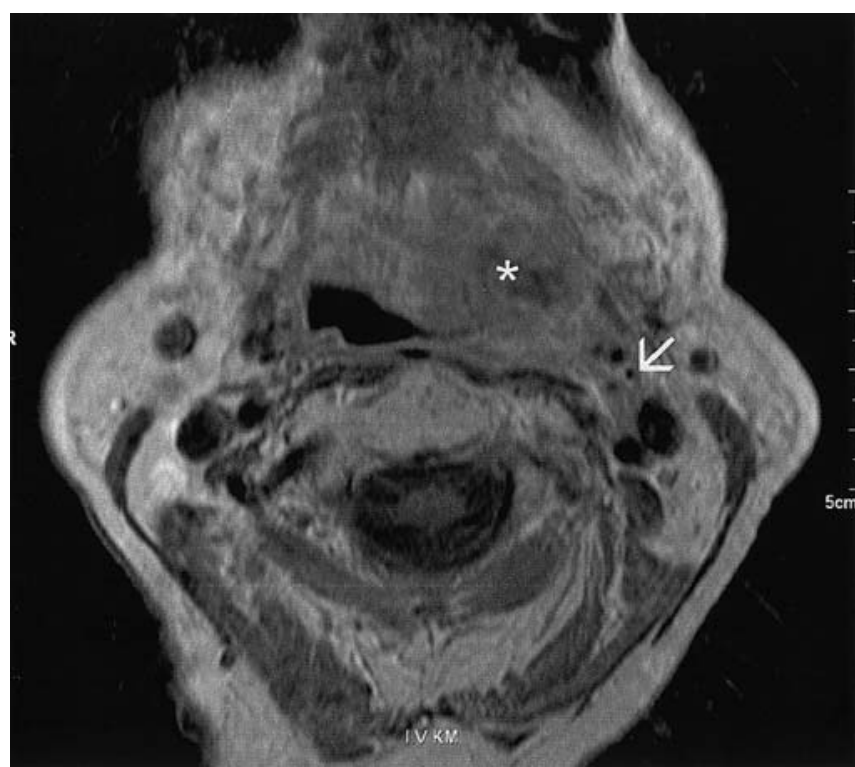

Fig. 3 Axial T1-weighted contrast-enhanced spin-echo MR image (TR/TE: $629 \mathrm{~ms} / 14 \mathrm{~ms}$ ) of a 71-year-old female patient suffering from referred pain. Left-sided large inhomogeneously enhancing soft tissue mass postero-laterally in the tongue base and adjacent tonsillar region (asterisk), showing direct tumor extension into the carotid space (arrow)

(Table 2), with a preponderance of higher tumor categories in patients with reflex otalgia. Most tumors were located in the tonsils or base of the tongue (Table 3 ).

The presence of reflex otalgia in regard to alterations of specific structures is demonstrated in Table 4, grouped according to their innervation by either the glossopharyngeal nerve (Table 4) or by other cranial nerves (Table 4). When reflex otalgia was present, structures innervated by the glossopharyngeal nerve were found to be significantly more often altered. Figures 2 and 3 show typical MRI images of two different patients suffering from reflex otalgia.

Infiltration of the uvula, the soft palate, base of the tongue, mucosal surface of the lateral and posterior pharyngeal wall, glossoepiglottic and pharyngoepiglottic folds and glossotonsillar sulcus was not significantly associated with reflex otalgia (Table 4).

Alteration of most anatomical regions and structures innervated by nerves other than the glossopharyngeal nerve showed no association with the clinical symptom of reflex otalgia. Figure 4 illustrates an MRI image of a patient with an oropharynx carcinoma without referred pain.

Of the structures not innervated by the glossopharyngeal nerve, the hard palate, the hyoglossus muscle and the preepiglottic space showed a significant association with the symptom of reflex otalgia (Table 4). Perineural spread of tumor was not observed in any patient on the MRI study. 


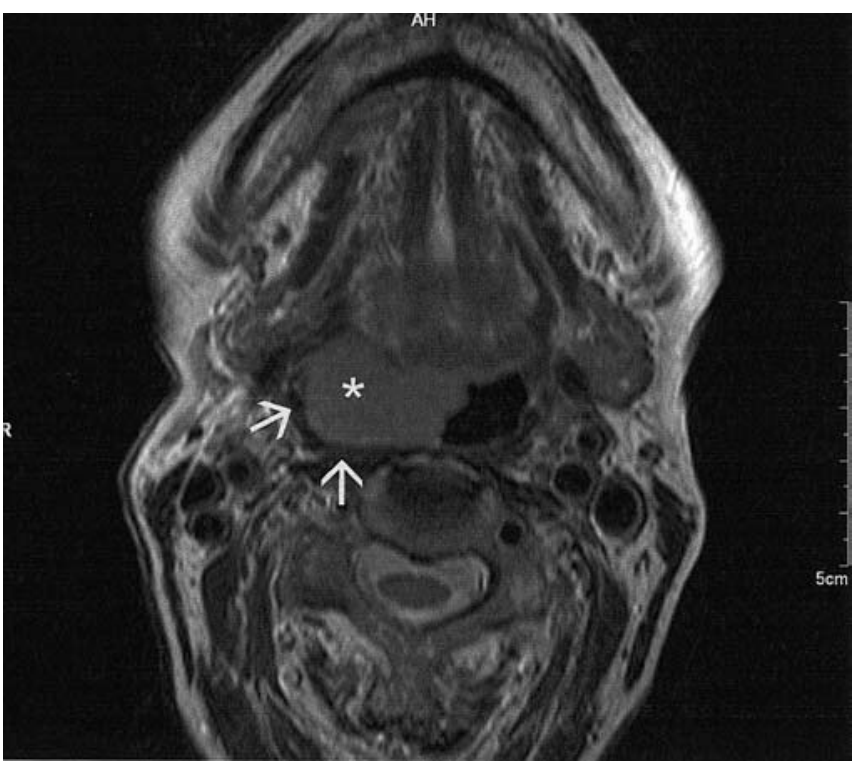

Fig. 4 Axial T2-weighted spin-echo MR image (TR/TE: 4,570 ms/ $125 \mathrm{~ms}$ ) of an 86-year-old female patient. This patient did not have ear pain. Well-delineated, soft tissue mass originating from the right tonsillar region corresponding to a squamous cell carcinoma (asterisk). Note that the superior constrictor pharyngis muscle is well preserved (arrows)

\section{Discussion}

The glossopharyngeal nerve, together with the trigeminal, facial and vagal nerves, is of branchial origin. These nerves all have sensory and motor neurons. The main sensory regions of the glossopharyngeal nerve are the mucosa of the middle ear, the palatine tonsils and soft palate, the nasopharynx, oropharynx and hypopharynx. The motor portion includes the superior constrictor pharyngis muscle and the stylopharyngeal, palatopharyngeal and palatoglossus muscles. Through the anastomosis with the tympanic nerve, a connection is established to the structures of the middle ear (Fig. 1). Therefore, irritation in the pharynx can be perceived as pain in the middle ear [1-4]. This important clinical symptom is known as referred pain, or reflex otalgia. Similar connections between different nerves to the nasopharynx and hypopharynx exist via the trigeminal, facial and vagal nerves. In order to evaluate a possible association between MRI alterations and the clinical symptom of referred pain in patients with oropharyngeal carcinoma, we analyzed the structures of the oropharynx and the adjacent regions as listed in Table 4.

Reflex otalgia or referred otalgia is a pain in the ear observed in various diseases of structures remote from the ear, and it is a clinical symptom well known to the ENT specialist. Head and neck cancers, but also benign lesions such as peritonsillar abscess or tonsillitis, can
Table 4 Infiltration of anatomical structures with regard to the clinical symptom of reflex otalgia

\begin{tabular}{lll}
\hline Structure altered & $\begin{array}{l}\text { Reflex } \\
\text { otalgia }\end{array}$ & $\begin{array}{l}\text { No reflex } \\
\text { otalgia }\end{array}$ \\
\hline
\end{tabular}

Structures innervated by the glossopharyngeal nerve

Nasopharynx

Soft palate

Uvula

Palatoglossus m.

Palatine tonsil

Palatopharyngeus $\mathrm{m}$.

Lateral pharyngeal wall

Superior constrictor pharyng. m.

Posterior pharyngeal wall

Retropharyngeal space

Stylopharyngeus $\mathrm{m}$.

Base of tongue

Glossotonsillar sulcus

Glossoepiglottic fold

Pharyngoepiglottic fold

Hypopharynx

$\begin{array}{rrl}9 & 2 & <0.003 \\ 9 & 8 & \text { n.s. } \\ 7 & 3 & \text { n.s. } \\ 14 & 7 & <0.001 \\ 15 & 12 & <0.022 \\ 9 & 4 & <0.038 \\ 13 & 10 & \text { n.s. } \\ 9 & 5 & <0.04 \\ 10 & 6 & \text { n.s. } \\ 3 & 0 & <0.036 \\ 7 & 1 & <0.006 \\ 13 & 10 & \text { n.s. } \\ 15 & 16 & \text { n.s. } \\ 8 & 8 & \text { n.s. } \\ 10 & 6 & \text { n.s. } \\ 7 & 3 & <0.045\end{array}$

Structures innervated by other cranial nerves

Base of skull

Levator veli palatini $\mathrm{m}$.

Tensor veli palatini $\mathrm{m}$.

Maxilla

Hard palate

Alveolar ridge

Prevertebral space

Parapharyngeal space

Retromolar trigone

Cheek

Masticator space

Intrinsic m. of tongue

Styloglossus m.

Hyoglossus m.

Genioglossus m.

Mylohyoid $\mathrm{m}$.

Geniohyoid $\mathrm{m}$

Digastricus m. ant. Belly

Digastricus m. post. Belly

Submandibular space

Sublingual space

Preepiglottic space

Epiglottis

Vocal cord

\begin{tabular}{lll}
1 & 0 & n.s. \\
2 & 0 & n.s. \\
2 & 0 & n.s. \\
1 & 0 & n.s. \\
3 & 0 & $<0.048$ \\
0 & 0 & n.s. \\
0 & 0 & n.s. \\
9 & 6 & n.s. \\
7 & 4 & n.s. \\
0 & 0 & n.s. \\
2 & 0 & n.s. \\
8 & 8 & n.s. \\
9 & 5 & n.s. \\
6 & 1 & $<0.002$ \\
6 & 3 & n.s. \\
0 & 0 & n.s. \\
0 & 0 & n.s. \\
0 & 0 & n.s. \\
0 & 1 & n.s. \\
2 & 0 & n.s. \\
1 & 1 & n.s. \\
5 & 1 & $<0.03$ \\
7 & 7 & n.s. \\
6 & 4 & n.s. \\
\hline
\end{tabular}

n.s., not significant

cause such referred pain $[1-6,10]$. The location of the disorder may thus be far more important than its etiology for this symptom. The pain perceived by the patient may be located in the middle ear (glossopharyngeal nerve), in the external auditory canal (vagal nerve) or in the periauricular region (trigeminal and facial nerves), due to the different pathways along the various cranial nerves.

In oropharynx carcinoma, the presence of referred otalgia is an important negative predictive and prognostic parameter for the probability of local control and disease-specific survival $[1,2]$. The authors showed that patients with reflex otalgia had a significantly higher rate 
of local recurrences after radical radiotherapy in comparison to patients without the symptom. Furthermore, disease-specific survival was markedly reduced if the referred pain was present. They postulate that reflex otalgia might indicate a more aggressive phenotype with a higher tendency to a more extensive infiltration in surrounding structures [1, 2]. To date, an association between the clinical symptom of referred pain and radiological findings of tumor infiltration of different anatomical structures has not been described. As it may prove to be of major clinical importance, we systematically analyzed the pretherapeutic MRI examinations in a consecutive series of patients with oropharynx carcinoma and correlated them to the specific structures involved to the clinical symptom of referred otalgia.

In accordance with previously published data, in our study no association of reflex otalgia was found to age, smoking or drinking habits or to tumor localization in the oropharynx $[1,2]$. The significant difference in gender distribution of reflex otalgia, with more female patients having this symptom, was not expected. It is unlikely that it represents a clinically important finding and may be explained by the low overall number of female patients. Since reflex otalgia is caused by local infiltration of the carcinomas into adjacent structures, it is evident that referred pain is found more often with bigger tumors and higher T-categories.

In patients suffering from reflex otalgia, structures innervated by the glossopharyngeal nerve were more often altered and probably infiltrated than in patients without this clinical symptom. Infiltration of the soft palate, the uvula, base of the tongue, mucosal surface of the posterior and lateral oropharyngeal wall, the glossoepiglottic and pharyngoepiglottic folds and the glossotonsillar sulcus was not significantly associated with reflex otalgia, despite their innervation by the glossopharyngeal nerve. Since we included only oropharynx carcinomas, obviously these structures were often infiltrated, no matter whether reflex otalgia was present or not.

A significant association of referred pain and an alteration of the hard palate, the preepiglottic space and the hyoglossus muscle were found. This is remarkable, since these structures are innervated by cranial nerves other than the glossopharyngeal nerve. One explanation might be the proximity to structures innervated by the glossopharyngeal nerve, individual variability in neural borders and the difficulty to define these borders. Reflex otalgia based on connections between the ear and the trigeminal, facial and glossopharyngeal nerves might be the likely pathophysiological mechanism in some of these patients with involvement of the nasopharynx or the hypopharynx. Furthermore, the majority of patients in this study were drinkers and smokers and had advanced tumors. Some of them may not have been able to localize the pain exactly, even when asked specifically. Others may have had combined glossopharyngeal, trigeminal, facial

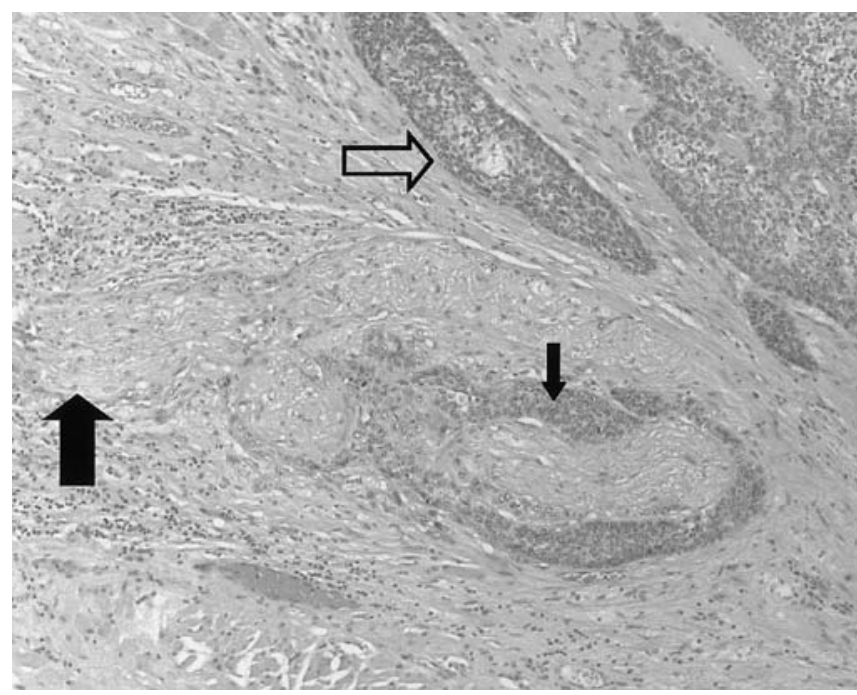

Fig. 5 Histological specimen of the lateral oropharynx wall of a 59-year-old male patient with reflex otalgia who had salvage surgery because of a recurrent squamous cell carcinoma (open arrow). The perineural infiltration of the carcinoma is shown by the small arrow; the large arrow delineates the corresponding nerve (hematoxylin-eosin stain; original magnification $\times 20$ )

and/or vagal nerve reflex otalgia. Finally, the low overall number of patients could pose a statistical problem.

Several shortcomings of this study have to be considered. First of all, distinction of individual peripheral and cranial nerves and their branches is usually not possible by MRI. Therefore, we had to examine anatomical structures innervated by specific nerves. Second, we did not observe any isolated associations between infiltration of a single anatomical structure and referred pain. It was nearly impossible to establish such an association, because different structures were usually infiltrated by the same tumor at the same time. Third, since differentiation by MRI was often impossible, we defined alteration both as infiltration by tumor and/or peritumoral edema. Although all tumors had been histologically proven, the biopsy site usually did not correspond to the structures evaluated in our study. Fourth, as the definitive treatment was radiotherapy or radiochemotherapy in all patients, only biopsies were available, and it was impossible to ascertain perineural spread from the histopathology report. Although we were unable to demonstrate perineural spread by MRI, we assume that perineural tumor infiltration was responsible for reflex otalgia in at least some patients. The anecdotal patient with recurrence and histological proof of perineural tumor infiltration at surgery would support this assumption (Fig. 5).

This suggests that there is an association between the alteration of structures innervated by the glossopharyngeal nerve on MRI and the clinical symptom of reflex otalgia. In case of a patient with ear pain and normal clinical and radiological findings of the ear and sur- 
rounding structures, disease remote from the ear causing referred pain must be ruled out $[3,4]$. Careful examination of the oropharynx and other anatomic areas supplied by branches of the glossopharyngeal, trigeminal, facial and vagus nerves is indicated.

\section{Conclusion}

In this study, an association was found between the clinical symptom of reflex otalgia and radiological (MRI) findings of alteration of structures innervated by the glossopharyngeal nerve in patients with oropharyngeal carcinoma. Except for the hard palate, the hyoglossus muscle, and the preepiglottic space, involvement of structures innervated by other cranial nerves did not show an association with referred pain.

Acknowledgements We thank E. Stauffer, MD, for the histologic slides, S.C. Marré, MD, for the data acquisition, and H. Mattle, $\mathrm{MD}$, for helpful comments.

\section{References}

1. Beer KT, von Briel C, Lampret T et al (1998) Predictive significance of reflex otalgia in local radical radiotherapy of oropharyngeal carcinomas. Strahlenther Onkol 174:306-310

2. Beer KT, Greiner RH, von Briel C, Thöni AF, Zbären P (1995) Reflex otalgia: prognostic relevance for radical radiotherapy of mesopharynx carcinomas. Eur J Cancer 31a [Suppl 5]:86

3. Weissman JL (1997) A pain in the ear: the radiology of otalgia. Am J Neuroradiol 18:1641-1651
4. Wazen JJ (1989) Referred otalgia. Otolaryngol Clin North Am 22:1205-1215

5. Thaller SR, De Silva A (1987) Otalgia with a normal ear. Am Fam Physician 36:129-136

6. Olsen KD (1986) The many causes of otalgia. Infection, trauma, cancer. Postgrad Med 80:50-63

7. Harnsberger HR (1995) Handbook of head and neck imaging, 2nd edn. Mosby, St. Louis

8. Leblanc A (1995) The cranial nerves: anatomy, imaging, vascularisation, 2nd edn. Springer, Berlin Heidelberg New York
9. Hermanek P, Hutter RVP, Sobin LH, Wagner G, Wittekind Ch (1997) TNM Atlas: illustrated guide to the TNM/pTNM classification of malignant tumours, 4th edn. Springer, Berlin Heidelberg New York

10. Smit M, Balm AJ, Hilgers FJ, Tan IB (2001) Pain as sign of recurrent disease in head and neck squamous cell carcinoma. Head Neck 23:372-375 